Erschienen in: Engelberg, Stefan/Holler, Anke/Proost, Kristel (Hrsg.):

Sprachliches Wissen zwischen Lexikon und Grammatik. - Berlin, Boston: de

Gruyter, 2011. S. 345-372. (Institut für Deutsche Sprache. Jahrbuch 2011), https://doi.org/10.1515/9783110262339.345

Joachim Jacobs

\title{
Grammatik ohne Wörter?
}

\begin{abstract}
Am Beispiel des Deutschen wird gezeigt, dass verschiedene Strategien, die traditionelle Unterscheidung zwischen Wörtern und Syntagmen so zu modifizieren, dass sie die sehr differenzierten Daten vollständig, detailliert und widerspruchsfrei erfasst, zur inhaltlichen Entleerung dieser Unterscheidung führen und sie damit letztlich überflüssig machen. Das gilt sowohl für die populäre Aufspaltung von Wort vs. Syntagma in mehrere spezifischere Kategorienpaare (phonologisches Wort vs. phonologisches Syntagma, graphematisches Wort vs. graphematisches Syntagma usw.) als auch für ihre Umdeutung als Prototypen- oder Default-Kategorien. Allerdings kann man an der Unterscheidung zwischen Wörtern und Syntagmen festhalten, wenn man Einschränkungen der Vollständigkeit, Detailtreue oder Widerspruchsfreiheit in Kauf nimmt (wie in der linguistischen Praxis oft unumgänglich). Diese Überlegungen übertragen sich auf die auf Wort vs. Syntagma beruhenden Abgrenzungen linguistischer Teilgebiete, wie Syntax vs. Morphologie vs. Phraseologie, z.T. auch Grammatik vs. Lexikon.
\end{abstract}

\section{Einleitung}

Das Verhältnis des Lexikons zur Grammatik ist nach gängigen Annahmen nicht nur durch den Gegensatz idiosynkratisch vs. regelhaft geprägt, sondern auch durch die Unterscheidung zwischen Wörtern und Syntagmen. Tatsächlich werden die beiden Gesichtspunkte \pm Regelhaftigkeit und \pm Wortstatus bei der Abgrenzung des Lexikons von der Grammatik oft nicht genau getrennt. Linguisten gehen häufig bequemerweise davon aus, dass das Lexikon als das Inventar der nicht vollständig regelhaften Einheiten aus Wörtern besteht - also eben der Wortschatz ist - und dass die Grammatik die Domäne der Syntagmen ist.

Deshalb erscheint es angebracht, im Rahmen einer Diskussion des Verhältnisses des Lexikons zur Grammatik noch einmal einen Blick auf die Unterscheidung von Wörtern und Syntagmen zu werfen, zumal diese ja weiteren umstrittenen Kompartmentalisierungen der Sprachwissenschaft

\footnotetext{
1 Für einsichtsvolle und anregende Kommentare danke ich den Teilnehmern der Jahrestagung des IDS. Viele interessante Hinweise erhielt ich auch an den Universitäten München und Tübingen, wo ich Vorfassungen des Textes vortragen durfte. Zu danken habe ich schließlich den Herausgebern dieses Bandes, die eine Reihe von nützlichen Verbesserungsvorschlägen gemacht haben. Nach Abschluss des Manuskripts brachte mir Martin Haspelmath seinen Aufsat $z$,The indeterminacy of word segmentation and the nature of morphology and syntax" zur Kenntnis, der in Folia Linguistica erscheinen wird. Haspelmath kommt aus allgemein-sprachwissenschaftlicher Sicht zu ähnlichen Ergebnissen wie ich. Leider konnte ich diese wichtige Arbeit hier nicht mehr berücksichtigen.
} 
zugrunde liegt, insbesondere der Einteilung in Morphologie (als Lehre von den komplexen Wörtern), Syntax (als Lehre von den Syntagmen) und Phraseologie (als Lehre von den verfestigten Syntagmen).

Dass die Trennung der Wörter von den Syntagmen schwierig ist, ist nicht neu. Es ist geradezu ein linguistischer Gemeinplatz, dass man keine klare Grenze zwischen diesen Ausdrucksstufen ziehen kann. In auffallendem Gegensatz dazu spielt jedoch die Unterscheidung zwischen Wörtern einerseits und Syntagmen oder Phrasen andererseits bis heute in der linguistischen Praxis und in vielen theoretischen Modellen eine zentrale Rolle. Das gilt sogar für konstruktionsgrammatische Ansätze, die, wie der von Croft (2001), nicht nur die Grenzziehung zwischen Grammatik und Lexikon, sondern auch viele andere traditionelle Einteilungen (etwa bei den Wortarten) in Frage stellen - aber eben nicht die Kategorien WORT und SYNTAGMA. ${ }^{2}$ Diese werden in Crofts Buch vielmehr als selbstverständlich vorausgesetzt. Eine andere repräsentative konstruktionsgrammatische Arbeit (Goldberg 2003) stellt sogar ausdrücklich fest, dass auch bei Aufhebung einer strikten Grenzziehung zwischen Lexikon und Grammatik ,the classical distinctions [nämlich zwischen Wörtern und Syntagmen, Anm. d. Verf.] retain their forcec (ebd., S. 119).

Es stellt sich aber die Frage, ob sie das wirklich tun. Ich möchte das hier, wenn auch mit Einschränkungen, verneinen. Ich werde zu zeigen versuchen, dass die einschlägigen Daten so differenziert sind, dass die Kategorien WORT und SYNTAGMA unter strengen wissenschaftlichen Standards nicht zu halten sind. Verschiedene Strategien, an ihnen festzuhalten, führen zu ihrer inhaltlichen Entleerung und damit dazu, dass sie überflüssig werden. - Am Ende will ich aber auch kurz deutlich machen, dass das Begriffspaar unter anderen Voraussetzungen durchaus brauchbar bleibt.

Insgesamt möchte ich also die theoretischen Konsequenzen der notorischen Abgrenzungsprobleme zwischen Wörtern und Syntagmen genauer herausarbeiten und dabei auch diese Probleme selbst noch einmal unter die Lupe nehmen, wobei ich mich auf Ergebnisse eines Wuppertaler DFGProjekts zur Grenzziehung zwischen Wörtern und Syntagmen stütze.

Den methodologischen Hintergrund bildet ein weiterer linguistischer Gemeinplatz, nämlich, dass die Brauchbarkeit einer Kategorisierung davon abhängt, was man mit ihr machen will. Wenn man also über Probleme der Unterscheidung von Wörtern und Syntagmen nachdenkt, sollte man sich zuerst klar machen, wozu man diese Unterscheidung verwenden will. Da gibt es mehrere Möglichkeiten, von denen ich hier nur eine, allerdings eine zentrale, genauer betrachte: Ich konzentriere mich auf die Rolle, die WORT und SYNTAGMA als metasprachliche Kategorien in einer empirisch adäquaten Beschreibung von Sprachsystemen spielen können. Dabei verstehe ich unter einer empirisch adäquaten Beschreibung des Systems einer Sprache L

Linguistische Kategorien schreibe ich in Großbuchstaben. 
eine, die sich zum Ziel setzt, die Ausdrücke von L vollständig und detailliert zu erfassen, und die sich dabei an allgemeinen wissenschaftsmethodologischen Standards, wie Widerspruchsfreiheit und Beschreibungsökonomie, orientiert. Diesem Konzept von empirischer Adäquatheit entspricht eine Norm, der sich viele Linguisten unterwerfen, indem sie bereit sind, Aussagen zu Sprachsystemen zu revidieren, wenn diese die Verhältnisse offensichtlich unvollständig, zu wenig detailliert, widersprüchlich oder unökonomisch wiedergeben. „Empirisch adäquate Sprachsystembeschreibung" kürze ich durch ,EAS ${ }^{c 6}$ ab.

\section{WORT und SYNTAGMA als reiche Kategorien}

Attraktiv, aber dann auch problematisch für EAS-Analysen sind WORT und SYNTAGMA vor allem deshalb, weil sie idealiter reiche Kategorien sind, also solche, aus deren Zutreffen sich eine große Zahl weiterer Eigenschaften vorhersagen lässt. So verbindet man mit der Kategorisierung eines sprachlichen Ausdrucks X als Wort die Erwartung, dass damit ein ganzes Cluster von Eigenschaften von X erfasst wird, anders als bei Kategorien wie STIMMHAFT oder MASKULINUM, die kaum mehr als eine einzige Eigenschaft festhalten. Dass solche reichen Kategorien in EAS-Zusammenhängen attraktiv sind, ergibt sich einerseits aus dem Streben nach detaillierter Beschreibung, andererseits aus dem Ziel, ökonomisch vorzugehen, erlauben es solche $\mathrm{Ka}$ tegorien doch, viele Fliegen mit einer Klappe zu schlagen. Bei den Kategorien WORT und SYNTAGMA kommt noch ein weiterer im EAS-Kontext wünschenswerter Zug dazu. Sie erfassen zusammen mit wenigen anderen Ausdrucksstufen (wie AFFIX) idealiter die Gesamtheit aller Ausdrücke der untersuchten Sprache: Wenn ein Ausdruck kein Wort ist, ist er, wenn er ,kleiner ${ }^{6}$ ist, ein Affix, wenn er größer ${ }^{6}$ ist, ein Syntagma.

Der Eindruck, man könne mit wenigen Stufenkategorien die Ausdrücke der jeweiligen Sprache vollständig abdecken und dabei gleichzeitig auf ökonomische Weise viele ihrer Details erfassen, ist wohl auch ein Grund dafür, dass man gerade diese Kategorien zur Definition ganzer Teilgebiete der Sprachbeschreibung - Morphologie, Syntax, Phraseologie - heranzieht. Zusätzlich manifestiert sich darin aber auch die Annahme, dass sich die Ausdrücke auf diesen verschiedenen Stufen so deutlich voneinander unterscheiden, dass es sich lohnt, sie separaten Teilgebieten zuzuweisen.

Konkretisieren wir das an den Stufenkategorien WORT und SYNTAGMA, so wie sie auf das Deutsche angewandt werden. Mit diesen Kategorien assoziieren Grammatiker des Deutschen mindestens die in dem Stufensystem 1 angegebenen Eigenschaften, wobei die Abkürzungen für die darunter folgenden Eigenschaftsdimensionen stehen: ${ }^{3}$

Die Eigenschaften sind in der Regel auf Wortformen, nicht auf Wortlexeme (also Zusammenfassungen von Wortformen mit gemeinsamen Eigenschaften) zu beziehen. 


\begin{tabular}{|l|c|c|}
\cline { 2 - 3 } \multicolumn{1}{c|}{} & WORT & SYNTAGMA \\
\hline DO-SYLB & + & - \\
\hline DO-PHONOTAKT & + & - \\
\hline DO-MINPHON & + & + \\
\hline INTERN-SPAT & - & + \\
\hline INTERN-MAJUSK & + & - \\
\hline DO-MINGRAPH & - & + \\
\hline FLEX-TEIL & + & - \\
\hline DERIV-BAS & + & - \\
\hline KOMP-BAS & - & + \\
\hline LK-TEIL & - & + \\
\hline VF-TEIL & - & + \\
\hline E-TEIL & & \\
\hline
\end{tabular}

Tab. 1: System 1
DO-SYLB:
Domäne für Syllabierung
DO-PHONOTAKT:
Domäne für die phonotaktischen Beschränkungen B1
DO-MINPHON:
Domäne für die phonologischen Minimalitätsbedingungen B2
INTERN-SPAT:
INTERN-MAJUSK:
Interne Spatien
DO-MINGRAPH:
Interne Majuskeln
Domäne für die graphematischen Minimalitätsbedingungen B3
FLEX-TEIL:
DERIV-BAS:
Separate Flexion von Teilgliedern
KOMP-BAS:
Basis für die Derivationsmuster M1
LK-TEIL:
Basis für die Kompositionsmuster M2
VF-TEIL:
In die linke Satzklammer bewegbares Teilglied
E-TEIL:
In das Vorfeld bewegbares Teilglied
Durch Modifikation, Komplementierung, Determination oder
Koordination erweiterbare Teilglieder

Die in System 1 angegebenen Werteverteilungen in diesen Eigenschaftsdimensionen entsprechen gängigen Annahmen über Unterschiede zwischen den Stufen: In den ersten drei Dimensionen kommt zum Ausdruck, dass Wörter eine andere lautliche Form haben als Syntagmen. Die nächsten drei halten Entsprechendes für die schriftliche Form fest. Es folgen drei Dimensionen, denen zufolge Flexion Teilausdrücke von Syntagmen, aber nicht von Wörtern betreffen kann und Derivations- und Kompositionsprozesse auf Wörtern, aber nicht auf Syntagmen operieren. Die Werteverteilungen in den folgenden drei Eigenschaftsdimensionen bringen zum Ausdruck, dass auch Bewegungs- und Erweiterungsprozesse keine Wortteile, sondern nur Teile von Syntagmen betreffen können. Diese Annahme ist unter der 
Bezeichnung „lexikalische Integrität“ populär (ein schöner Beleg für die eingangs erwähnte Tendenz, die Begriffe, lexikalisch` und ,auf Wörter bezogen $^{\circ}$ gleichzusetzen).

Jedem der vier Blöcke ließen sich weitere Eigenschaftsdimensionen hinzufügen, dem letzten etwa die Möglichkeit weiterer Dislozierungprozesse oder das Auftreten interner Rektions- oder Kongruenzbeziehungen. Es fehlen auch Eigenschaften, die zu keinem der Blöcke gehören, wie die lexikalische Reihenbildung, ${ }^{4}$ die in ausgeprägter Form nur bei Wörtern, nicht bei Syntagmen aufzutreten scheint. Auch semantische Eigenschaften, die als diagnostisch für die Unterscheidung von Wörtern und Syntagmen betrachtet werden, fehlen in der Liste, etwa die Referentialität von Teilgliedern. Und schließlich verzeichnet die Liste keine Eigenschaften, durch die sich typische Wörter und Syntagmen nicht voneinander, sondern gemeinsam von anderen Stufen, insbesondere von Affixen, unterscheiden. Dazu gehört die variable Stellung im Satz.

Dennoch ist die Liste umfangreich genug, um an ihr Glanz und Elend der Stufenkategorien sichtbar zu machen. Zunächst zum Glanz: Man sieht sofort, dass die Kategorien WORT und SYNTAGMA tatsächlich eine Fülle von Eigenschaften ihrer Elemente festhalten. Und weil die vielen Eigenschaftsdimensionen je gegensätzliche Werte annehmen, werden die Elemente der Kategorien als deutlich verschieden beschrieben, wie es sein sollte, wenn man Wörtern und Syntagmen eigene Teilgebiete der Sprachwissenschaft widmen will. Dafür, dass sich eine solche Gebietstrennung lohnt, spricht auch, dass jede der beiden Kategorien anscheinend eine riesige Menge von Ausdrücken umfasst, was in (1) nur an ganz wenigen Beispielen angedeutet ist:
a. WORT: sprechen, spricht, besprechen, Sprache, ...
b. SYNTAGMA: laut sprechen, mit Gerda sprechen, lebendige Sprache, ...

Man erkennt aber auch gleich Einschränkungen. So ist klar, dass die Kategorien WORT und SYNTAGMA, wie sie hier spezifiziert sind, nicht auf alle Sprachen übertragbar sind. Viele Sprachen haben ja kein Schriftsystem mit Spatien oder gar mit satzinternen Majuskeln, und auch die linke Satzklammer und das Vorfeld sind spezifisch deutsch. Die in der allgemeinen Sprachwissenschaft lang diskutierte Frage, ob die Kategorien WORT und SYNTAGMA universell sind, muss also hinsichtlich der konstitutiven Eigenschaftsdimensionen - und erst recht im Hinblick auf die konkreten Eigenschaften - verneint werden. Allenfalls gibt es Universalität auf der Ebene der Blöcke, zu denen wir die Dimensionen zusammengefasst haben.

Darunter verstehe ich die Aufnahme einer größeren Zahl von nach demselben Muster gebildeten Ausdrücken in den Bestand der usualisierten Ausdrücke der jeweiligen Sprache. 
Vielleicht haben also alle Sprachen Wörter im Sinn von Einheiten mit einer sprachspezifischen lautlichen und gegebenenfalls schriftlichen Form, die $\mathrm{Ba}-$ sis für die in der Sprache möglichen morphologischen Prozesse sind, aber den möglichen Umstellungs- und Erweiterungsprozessen den Zugang verwehren, und die sich durch diese Eigenschaften von anderen Einheiten, eben denen, die in der Sprache als Syntagmen gelten, unterscheiden. ${ }^{5}$ Diese Relativierung auf die Spezifika einzelner Sprachen impliziert, dass das Wort in Sprachen, in denen nicht alle Eigenschaftsdimensionen einschlägig sind, blassere Konturen hat. So ist das Wort im Chinesischen, das keine Schrift mit Worttrennungszeichen und kaum morphologische Prozesse hat, wohl keine sehr prägnante Einheit. ${ }^{6}$

Das Problem fehlender Einschlägigkeit von Stufenkriterien, wie ich die Eigenschaftsdimensionen auch nenne, manifestiert sich aber nicht nur im Sprachvergleich, sondern auch in der Analyse von Einzelsprachen. Mehrere unserer Kriterien lassen sich ja nicht auf alle Ausdrucksklassen des Deutschen anwenden: Das Majuskelkriterium läuft leer, wenn es keine nominalen Teilausdrücke gibt, das Flexionskriterium kann man nicht auf Partikeln anwenden, die Bewegungskriterien sind nur für verbale Projektionen einschlägig, usw. Wie geht man z.B. mit einem Ausdruck wie sehr gern um, für den, weil er nur aus Partikeln besteht, weder das Majuskelkriterium noch das Flexionskriterium, noch die Bewegungskriterien einschlägig sind? Üblicherweise zieht man in solchen Fällen nur die einschlägigen Kriterien in Betracht, hier etwa das Spatienkriterium und die phonologischen Kriterien, nach denen sehr gern ein Syntagma ist. Um keine zu exotische Position einzunehmen, schließe ich mich dieser Praxis an. Festzuhalten ist aber, dass die Kategorien WORT und SYNTAGMA infolge der Nicht-Einschlägigkeit von Kriterien bei vielen Ausdrücken nicht so viele Eigenschaften voraussagen, wie man das angesichts der umfangreichen Liste in Tabelle 1 zunächst erwarten würde.

\section{Abweichungen vom Idealfall und die Aufspaltung des Stufensystems}

Doch auch, wenn man nur die je einschlägigen Kriterien betrachtet, findet man häufig nicht die idealen Werteverteilungen aus System 1. Ich betrachte nun einige Hauptgruppen dieser nicht-idealen Fälle und stelle gleichzeitig eine naheliegende und (bis zu einem bestimmten Punkt) populäre Strategie des Umgangs mit solchen Fällen vor.

Einen guten Überblick über den heutigen Stand der allgemein-sprachwissenschaftlichen Diskussion über den Status der Kategorie WORT gibt Dixon/Aikhenvald (Hg:) (2002).

Tatsächlich ist für das Chinesische bestritten worden, dass es überhaupt Wörter hat, vgl. z.B. Hockett (1944). 
3.1 Abweichungen im Hinblick auf phonologische Kriterien

Viele Ausdrücke weichen in phonologischen Stufenkriterien vom Idealfall ab. Dazu gehören sämtliche Komposita. Diese verhalten sich in der Fuge hinsichtlich der Syllabierung und oft auch phonotaktisch so, wie es von Syntagmen zu erwarten wäre, obwohl sie nach vielen anderen Kriterien Wörter sind, vgl. im Hinblick auf die Syllabierung z.B. [blu:t.ro:t] vs. *[blus.tso:t] blutrot, im Hinblick auf die Phonotaktik z.B. [ba:n.kundə] vs. *[ba:ı.kundə] Babnkunde?

Das Problem ist bekannt, und bekannt ist auch eine bestimmte Lösung: Man spaltet von den ursprünglichen Stufenkategorien neue Kategorien ab, in denen die phonologischen Kriterien von den anderen Kriterien getrennt werden. Mit diesen neu definierten Stufen PHONOLOGISCHES WORT und PHONOLOGISCHES SYNTAGMA (P-WORT bzw. P-SYNTAGMA) kann unser Stufensystem wie in Tabelle 2 erweitert werden:

\begin{tabular}{|l|c|c|}
\cline { 2 - 3 } \multicolumn{1}{c|}{} & WORT & SYNTAGMA \\
\hline INTERN-SPAT & - & + \\
\hline INTERN-MAJUSK & - & + \\
\hline DO-MINGRAPH & + & + \\
\hline FLEX-TEIL & - & - \\
\hline DERIV-BAS & + & - \\
\hline KOMP-BAS & + & + \\
\hline LK-TEIL & - & + \\
\hline VF-TEIL & - & + \\
\hline E-TEIL & - & - \\
\hline
\end{tabular}

\begin{tabular}{|l|c|c|}
\cline { 2 - 3 } \multicolumn{1}{c|}{} & P-WORT & P-SYNTAGMA \\
\hline DO-SYLB & + & - \\
\hline DO-PHONOTAKT & + & - \\
\hline DO-MINPHON & + & - \\
\hline
\end{tabular}

Tab. 2: System 2

Ein wichtiges Merkmal dieses Kategorisierungssystems ist, dass die neuen mit den ursprünglichen Stufen widerspruchsfrei kombinierbar sind, da die beiden Kategorienpaare auf unterschiedlichen Kriterien beruhen. Das System erlaubt also Kreuzklassifikationen wie in (2):

Auch viele deutsche Präfix-Bildungen verhalten sich phonologisch wie Syntagmen, nach den meisten anderen Kriterien aber wie Wörter. 
(2) a. WORT, P-WORT: sprechen, befragen, Frage, ...

b. WORT, P-SYNTAGMA: Babnkunde, blutrot, ...

c. SYNTAGMA, P-SYNTAGMA: schnell sprechen, Gerda befragen, viele schwierige Fragen, ...

Die Vorteile dieser Lösung, die sie so beliebt gemacht haben, liegen auf der Hand: Man kann, soweit bisher erkennbar, nun auch die Problemfälle vollständig und widerspruchsfrei einordnen und dabei ihre Eigenschaften detailliert erfassen.

Für diese Vorteile von System 2 muss man allerdings eine Verarmung der einzelnen Stufen in Kauf nehmen. Jede der Stufen in System 2 sagt weniger Eigenschaften voraus als die beiden ursprünglichen Stufen in System 1.

Diese geringere Prädiktivität der Stufen hat Linguisten aber kaum zu denken gegeben, denn dass man für lautliche Zusammenhänge separate Kategorien braucht, erscheint ganz normal. Tatsächlich wird die Kategorie P-WORT oft völlig losgelöst von der ursprünglichen Kategorie WORT betrachtet, nämlich als rein prosodische Einheit, die hierarchisch über den prosodischen Einheiten Silbe und Fuß steht. ${ }^{8}$ Aus dieser Sicht ist allerdings kaum erklärlich, warum die Grenzen von P-Wörtern immer Morphemgrenzen sind, ${ }^{9}$ während Silben und Füße nicht mit inhaltlichen Grenzen koinzidieren müssen. Wenn man dagegen im Blick behält, dass das P-Wort aus dem ursprünglichen Wortbegriff abgespalten wurde, um bestimmte nicht-ideale Fälle zu erfassen, versteht man das: Die Grenzen von Wörtern - seien sie nun ideal oder nicht - sind ja stets Morphemgrenzen (weil Wörter auf jeden Fall Ausdrücke sind, also aus mindestens einem Morphem bestehen). ${ }^{10}$

Ein weiterer unschöner Zug des erweiterten Stufensystems ist, dass die phonologischen Eigenschaften, die das P-Wort nach System 2 definieren, manchmal nicht in der vorgesehenen Kombination auftauchen. So verhalten sich schwache Formen vieler Funktionswörter hinsichtlich der Syllabierung wie P-Wörter, im Hinblick auf Minimalitätsbedingungen jedoch nicht: Es heißt [n.?i:gal] ' $n$ Igelund nicht [ni:.gal], der schwache Artikel ist also eine eigene Syllabierungsdomäne. Sein Umfang erreicht jedoch nicht das für P-Wörter (nach Hall 1999b) erforderliche Minimum einer zweimorigen Silbe.

Vgl. z.B. Hall (1999a).

Ebd., S. 2.

Auf die gleiche Weise kann man erklären, dass auch die Grenzen von P-Syntagmen stets mit Morphemgrenzen koinzidieren. 
Dieses Problem ist Phonologen durchaus bekannt, ${ }^{11}$ es gibt aber keine allgemein akzeptierte Lösung. Manche Forscher ordnen problematische Fälle wie die schwachen Funktionswörter gar keiner Stufe mehr zu, ${ }^{12}$ geben also die Idee preis, dass das Stufensystem die Ausdrücke der Sprache vollständig abdecken soll. Andere führen für die Problemfälle zusätzliche Stufen ein, etwa P-KLITIKON, ${ }^{13}$ womit sie Vollständigkeit sichern, aber die einzelnen Stufen in ihrer Aussagekraft weiter reduzieren.

\subsection{Abweichungen im Hinblick auf graphematische Kriterien}

Probleme dieser Art treten auch auf der graphematischen Ebene auf. Divergenzen zwischen dem Spatienkriterium und anderen Stufenkriterien sind schon lange bemerkt, aber erst vor kurzem detailliert analysiert worden. ${ }^{14}$ Sie betreffen u.a. die phonologischen Kriterien: $\mathrm{Ob}$ wir zwischen zwei Ausdrücke ein Spatium setzen, ist weitgehend unabhängig davon, ob zwischen ihnen eine P-Wort-Grenze liegt. Oft fehlen Spatien, wo es P-Wort-Grenzen gibt, so bei sämtlichen Komposita, und manchmal setzen wir ein Spatium, wo nur einer der separierten Ausdrücke ein zweifelsfreies P-Wort ist, so im erwähnten Beispiel <'n Igel $>$ (vs. ${ }^{*}<$ 'nigel $>$ ). Aber auch vielen nicht-phonologischen Kriterien läuft die Spatiensetzung zuwider, etwa den Bewegungskriterien. So schreiben wir Partikelverben ohne Spatium, obwohl ihre Teile durch Bewegung in die linke Satzklammer getrennt werden können, z.B. $<$ abspricht $>$ vs. $*<$ ab spricht $>$.

Diese vielen Divergenzen beruhen darauf, dass die deutsche Spatiensetzung, so wie sie sich historisch herausgebildet hat, bei weitem nicht für alle anderen Wortkriterien sensitiv ist. Das gilt besonders für die Getrenntschreibung, die überhaupt nicht auf Wortkriterien beruht, sondern auf dem Prinzip, dass zwischen Ausdrücken, also bedeutungshaltigen Abschnitten aller Art, ein Spatium zu setzen ist. Dieses Prinzip wird nur dann durch Zusammenschreibung von Ausdrücken verletzt, wenn deren Verbindung sich nach einigen wenigen Kriterien wie ideale komplexe Wörter verhält, etwa nach den morphologischen Kriterien. Für viele andere Kriterien, etwa die phonologischen oder die Bewegungskriterien, ist die Zusammenschreibung dagegen völlig blind. ${ }^{15}$

\footnotetext{
Vgl. z.B. Hall (1999a), Raffelsiefen (1999).

12 So Hall (1999b), bei dem die schwachen (und auch die starken) Formen von Funktionswörtern direkt von einer höheren prosodischen Stufe (der P-Phrase, entspricht unserem P-Syntagma) dominiert werden, ohne selbst einer Stufe zugeordnet zu werden.

Vgl. z.B. Nespor/Vogel (1986).

Vgl. Jacobs (2005), Fuhrhop (2007).

15 Vgl. Jacobs (2005, Kap. 4.1.4.). Im Lichte dieser Überlegungen ist der Behauptung von Nübling et al. (2008) zu widersprechen, dass es in der Geschichte des deutschen Schriftsystems Entwicklungen gegeben habe, die dazu beitrugen „dass die Grenzen des phonologischen Worts auch in der Schrift erkennbar sind“ (ebd., S. 40). Nübling et al. erwähnen in diesem
} 
Was nun die daraus resultierenden Probleme für das Stufensystem betrifft, gehen viele Linguisten von einer Lösung aus, wie wir sie schon bei den phonologischen Kriterien gesehen haben: Man lagert die graphematischen Kriterien in ein zusätzliches Stufenpaar GRAPHEMATISCHES WORT vs. GRAPHEMATISCHES SYNTAGMA (G-WORT bZw. G-SYNTAGMA) aus, so wie in System 3:16

\begin{tabular}{|l|c|c|}
\cline { 2 - 3 } \multicolumn{1}{c|}{} & WORT & SYNTAGMA \\
\hline FLEX-TEIL & - & + \\
\hline DERIV-BAS & + & - \\
\hline KOMP-BAS & + & + \\
\hline LK-TEIL & - & + \\
\hline VF-TEIL & - & + \\
\hline E-TEIL & - & + \\
\hline
\end{tabular}

\begin{tabular}{|l|c|c|}
\cline { 2 - 3 } \multicolumn{1}{c|}{} & P-WORT & P-SYNTAGMA \\
\hline DO-SYLB & + & - \\
\hline DO-PHONOTAKT & + & - \\
\hline DO-MINPHON & + & - \\
\hline
\end{tabular}

\begin{tabular}{|l|c|c|}
\cline { 2 - 3 } \multicolumn{1}{c|}{} & G-WORT & G-SYNTAGMA \\
\hline INTERN-SPAT & - & + \\
\hline INTERN-MAJUSK & - & + \\
\hline DO-MINGRAPH & + & - \\
\hline
\end{tabular}

Tab. 3: System 3

Diese Lösung hat die gleichen Vorteile wie die Einführung des P-Worts: Die mit ihr mögliche Kreuzklassifikation, wie in (3), scheint eine widerspruchsfreie und vollständige Erfassung auch der problematischen Fälle zu ermöglichen und hält deren Eigenschaften genau fest:

Zusammenhang allerdings nicht die Spatiensetzung, sondern die Konstantschreibung von Morphemen und die Substantivgroßschreibung. Beide Phänomene haben jedoch wenig mit phonologischen Wörtern zu tun. (Wenn es in der Spatiensetzung einen historischen Trend zur besseren Kennzeichnung von P-Wörtern gegeben hätte, wäre u.a. der Aufbau einer konsequenten Getrenntschreibung von Kompositionsgliedern und Präfixen zu erwarten gewesen. Faktisch ist jedoch gerade die Zusammenschreibung solcher Elemente gestärkt worden.)

16 Eine repräsentative Arbeit zum graphematischen Wort ist Fuhrhop (2008). 
(3) a. WORT, P-WORT, G-WORT: sprechen, befragen, Frage, ...

b. WORT, P-SYNTAGMA, G-WORT: Bahnkunde, blutrot, ...

c. SYNTAGMA, ${ }^{17}$ P-SYNTAGMA, G-WORT: absprechen, aussprechen, ...

d. SYNTAGMA, P-SYNTAGMA, G-SYNTAGMA: schnell sprechen, Gerda befragen, viele schwierige Fragen, ...

System 3 geht aber auch mit einer weiteren Verarmung der Stufen einher. Vor allem die Basisstufen WORT und SYNTAGMA sagen nun noch weniger Eigenschaften voraus als in System 2. Auch hier könnte man sich aber damit trösten, dass das Schriftsystem eine eigene Repräsentationsebene ist, die eigene Kategorien erfordert, wie das für die graphematische Silbe etwa in Primus (2003) gezeigt wurde.

Leider erfassen die neuen Stufen aber wieder nicht alle Fälle. Divergenzen zwischen graphematischen Wortkriterien gibt es z.B. bei Akronymen wie $\langle\mathrm{DD}\rangle,\langle\mathrm{DFG}\rangle,\langle\mathrm{SPD}\rangle$. Nach dem Spatienkriterium sind sie G-Wörter, nach dem Majuskelkriterium nicht, und sie verletzen auch die Bedingung, dass G-Wörter (nach Fuhrhop 2008) minimal aus einer graphematischen Vollsilbe bestehen. Um angesichts solcher Fälle eine vollständige und widerspruchsfreie Stufenzuordnung zu sichern, bietet es sich an, die Kategorie G-WORT weiter aufzuspalten, aber das führt dann zu einer noch weiteren Verarmung der einzelnen Stufen.

\subsection{Abweichungen im Hinblick auf morphologische Kriterien}

Eine weitere Verarmung ist aber bei Beibehaltung der Aufspaltungsstrategie ohnehin nicht zu vermeiden, wie die vielfältigen Probleme mit den Kriterien FLEX-TEIL, DERIV-BAS und KOMP-BAS zeigen. So gibt es separate Flexion von Teilgliedern nicht nur bei eindeutigen Syntagmen, sondern auch bei Ausdrücken, die nach vielen anderen Kriterien Wörter sind, etwa bei $\mathrm{V}+\mathrm{V}$-Komposita, wie gefriertrocknen oder rïhrbraten, bei denen das Partizip-IIPräfix ge- und die Infinitiv-Markierung $q u$ wie bei verbalen Syntagmen am Zweitglied ansetzen, vgl. gefrierqutrocknen vs. ?? qu gefriertrocknen, rührgebraten vs. * gerïbrbraten. Noch bekanntere Beispiele für wortartige Ausdrücke mit separater Flexion von Teilen (also mit Binnenflexion) findet man unter den Zusammenrückungen, wie der Hohepriester, die Hohenpriester.

Dieses Phänomen kann man nicht auf fehlenden phonologischen Wortstatus zurückführen. Zwar sind bei separater Flexion meist mehrere phonologische Wörter im Spiel, aber bei weitem nicht alle Ausdrücke, die mehr als

17 Die Einordnung von Partikelverben als Syntagmen wird noch zu überdenken sein, siehe unten. 
ein phonologisches Wort umfassen, zeigen separate Flexion. So werden Präfixverben, auch wenn sie aus zwei phonologischen Wörtern bestehen, als ganze flektiert, vgl. zu überlassen vs. * ̈̈berqulassen.

Es bleibt also im Rahmen der bisher angewandten Strategie nichts anderes übrig, als ein weiteres Stufenpaar FLEXIONSMORPHOLOGISCHES WORT vs. FLEXIONSMORPHOLOGISCHES SYNTAGMA (F-WORT bZW. F-SYNTAGMA) anzunehmen, wie in System 4:

\begin{tabular}{|l|c|c|}
\cline { 2 - 3 } \multicolumn{1}{c|}{} & WORT & SYNTAGMA \\
\hline DERIV-BAS & + & - \\
\hline KOMP-BAS & + & - \\
\hline LK-TEIL & - & + \\
\hline VF-TEIL & - & + \\
\hline E-TEIL & - & + \\
\hline
\end{tabular}

\begin{tabular}{|l|c|c|}
\cline { 2 - 3 } \multicolumn{1}{c|}{} & P-WORT & P-SYNTAGMA \\
\hline DO-SYLB & + & - \\
\hline DO-PHONOTAKT & + & - \\
\hline DO-MINPHON & + & - \\
\hline
\end{tabular}

\begin{tabular}{|l|c|c|}
\cline { 2 - 3 } \multicolumn{1}{c|}{} & G-WORT & G-SYNTAGMA \\
\hline INTERN-SPAT & - & + \\
\hline INTERN-MAJUSK & - & + \\
\hline DO-MINGRAPH & + & - \\
\hline
\end{tabular}

\begin{tabular}{|l|c|c|}
\cline { 2 - 3 } \multicolumn{1}{c|}{} & F-WORT & F-SYNTAGMA \\
\hline FLEX-TEIL & - & + \\
\hline
\end{tabular}

Tab. 4: System 4

In dem System in Tabelle 4 bringen wir unsere Problemfälle unter, wie (4) andeutet:

(4) a. WORT, P-WORT, G-WORT, F-WORT: sprechen, befragen, Frage,...

b. WORT, P-SYNTAGMA, G-WORT, F-WORT:

Bahnkunde, blutrot, ...

c. WORT, P-SYNTAGMA, G-WORT, F-SYNTAGMA:

rübrbraten, Hohepriester, ... 
d. SYNTAGMA, P-SYNTAGMA, G-WORT, F-SYNTAGMA: absprechen, aussprechen, ...

e. SYNTAGMA, P-SYNTAGMA, G-SYNTAGMA, F-SYNTAGMA: schnell sprechen, Gerda befragen, viele schwierige Fragen, ...

Aber natürlich zahlen wir wieder den Preis einer weiteren Verarmung einzelner Stufen, hier schon bis zu dem Extrem, dass ein Stufenpaar, eben F-WORT vs. F-SYNTAGMA, nur noch eine einzige Eigenschaft voraussagt.

Vielleicht ist das ein Grund dafür, dass das F-WORT als eigene Stufe zwar tatsächlich vorgeschlagen wurde, ${ }^{18}$ sich aber, anders als das P- und das G-WORT, nicht durchgesetzt hat. Dabei dürfte auch eine Rolle gespielt haben, dass sich die Flexionsmorphologie im Gegensatz zur Phonologie und zur Graphematik kaum als separate Repräsentationsebene konzipieren lässt, für die ohnehin eigene Kategorien anzunehmen sind. (Flexionsmorphologische Kategorien sind ja nach üblichem Verständnis eng verwoben mit der Syntax und auch mit anderen morphologischen Kategorien.)

Man kann das Problem auch nicht dadurch verkleinern, dass man statt der bettelarmen Kategorie des F-WORTS eine etwas reichere annimmt, die wenigstens noch die anderen morphologischen Kriterien, DERIV-BAS und KOMP-BAS, umfasst. Dagegen sprechen die Daten: Als Basen für einschlägige Derivations- und Kompositionsprozesse können auch Ausdrücke mit separat flektierten Teilen dienen, etwa viele Partikelverben, die z.B. bei der Adjektivierung mit -bar einen großen Teil der Basen ausmachen, vgl. anfechtbar, auffindbar, einstellbar.

Dieses Beispiel zeigt im Übrigen, dass die stammbildungsmorphologischen Kriterien nicht nur mit dem flexionsmorphologischen, sondern auch mit syntaktischen Stufenkriterien konfligieren können, denn Partikelverben sind auch nach Bewegungskriterien Syntagmen. Also müssen wir auch die stammbildungsmorphologischen Kriterien von den syntaktischen trennen und bei Beibehaltung der bisherigen Strategie für sie ein weiteres spezielles Stufenpaar einführen. Damit erhalten wir das Stufensystem in Tabelle 5 und die in (5) illustrierte Kreuzklassifikation.

Und wieder sinkt die Aussagekraft der einzelnen Stufen. Dass die neuen Stufen WB-WORT und WB-SYNTAGMA nur geringes prädiktives Potenzial haben, hat im Übrigen einen weiteren Grund, der in der einleitenden Formulierung der entsprechenden Stufenkriterien schon angedeutet ist: Nicht alle Derivationsmuster sind sensitiv dafür, ob ihre Basen Worteigenschaften haben. Es gibt solche wie die -bar-Adjektivierung, die tatsächlich außer Partikelverben nichts als Basis akzeptieren, was deutliche Züge eines Syntagmas hat. Selbst Inkorporationsbildungen, die sonst den Partikelverben ähneln, können nach Untersuchungen in dem erwähnten Wuppertaler Forschungsprojekt kaum adjektiviert werden, z.B. ??bloßstellbar, ?? kennenlembar,

18 So in Wurzel (2000). 
*gefangennehmbar ${ }^{19}$ Es gibt aber auch Muster wie die -er-Nominalisierung, die bereitwillig Syntagmen als Basen akzeptieren, man denke an bekannte Beispiele wie Schwarsseher oder Wichtigtuer, bei denen man die Annahme eines Syntagmas als Basis nur durch die Ad-hoc-Postulierung einer vorgängigen Konversion des Zweitglieds vermeiden kann.

\begin{tabular}{|l|c|c|}
\cline { 2 - 3 } \multicolumn{1}{c|}{} & WORT & SYNTAGMA \\
\hline LK-TEIL & - & + \\
\hline VF-TEIL & - & + \\
\hline E-TEIL & - & + \\
\hline
\end{tabular}

\begin{tabular}{|l|c|c|}
\cline { 2 - 3 } \multicolumn{1}{c|}{} & P-WORT & P-SYNTAGMA \\
\hline DO-SYLB & + & - \\
\hline DO-PHONOTAKT & + & - \\
\hline DO-MINPHON & + & - \\
\hline
\end{tabular}

\begin{tabular}{|l|c|c|}
\cline { 2 - 3 } \multicolumn{1}{c|}{} & G-WORT & G-SYNTAGMA \\
\hline INTERN-SPAT & - & + \\
\hline INTERN-MAJUSK & - & + \\
\hline DO-MTNGRAPH & + & - \\
\hline
\end{tabular}

\begin{tabular}{|c|c|c|}
\cline { 2 - 3 } \multicolumn{1}{c|}{} & F-WORT & F-SYNTAGMA \\
\hline FLEX-TEIL & - & + \\
\hline
\end{tabular}

\begin{tabular}{|l|c|c|}
\cline { 2 - 3 } \multicolumn{1}{c|}{} & WB-WORT & WB-SYNTAGMA \\
\hline DERIV-BAS & + & - \\
\hline KOMP-BAS & + & - \\
\hline
\end{tabular}

Tab. 5: System 5

(5) a. WORT, P-WORT, G-WORT, F-WORT, WB-WORT: sprechen, befragen, Frage, ...

b. WORT, P-SYNTAGMA, G-WORT, F-WORT, WB-WORT: Babnkunde, blutrot, ...

c. WORT, P-SYNTAGMA, G-WORT, F-SYNTAGMA, WB-WORT:20 rïbrbraten, Hohepriester, ...

19 Entsprechende Beispiele wurden von kaum mehr als $30 \%$ unserer Informanten für sprachlich richtig gehalten, im Gegensatz zu Partikelverben mit -bar (wie vorlesbar), die über $80 \%$ erreichten.

20 Die Zuordnung der genannten Beispiele zur Kategorie WB-WORT ist nicht ganz sicher. Dazu müsste genauer geprüft werden, bei welchen Derivations- und Kompositionsprozessen die Beispiele als Basen fungieren können. 
d. SYNTAGMA, P-SYNTAGMA, G-WORT, F-SYNTAGMA, WB-WORT:

absprechen, aussprechen, ...

e. SYNTAGMA, P-SYNTAGMA, G-WORT, F-SYNTAGMA, WB-SYNTAGMA:

gefangen nebmen, kennenlernen, ...

f. SYNTAGMA, P-SYNTAGMA, G-SYNTAGMA, F-SYNTAGMA, WB-SYNTAGMA:

schnell sprechen, Gerda befragen, viele schwierige Fragen, ...

Deshalb habe ich oben das Kriterium DERIV-BAS nicht als Basisfähigkeit für Derivation allgemein, sondern als Basisfähigkeit für bestimmte Derivationsmuster formuliert, die, wie die -bar-Adjektivierung, sensitiv für Stufenkriterien sind.

Die gleiche Einschränkung gilt für die Komposition und das Kriterium KOMP-BAS. Manche Kompositionsmuster akzeptieren tatsächlich keine Basen mit Merkmalen von Syntagmen. Dazu gehören alle koordinativen Muster, vgl. Dichter-Komponist vs. * Gefeierter-Dichter-Komponist (vs. gefeierter DichterKomponist). Andere Kompositionsmuster lassen dagegen für ihr Erstglied solche Basen zu, wie die deswegen so genannte Phrasenkomposition, z.B. Graue-Schläfen-Effekt, Trimm-dich-Pfad. ${ }^{21}$ Syntagmen als Basen kommen aber z.B. auch bei der Verbstamm+N-Komposition vor, z.B. Kennenlem-Aktion, Schnellsprech-Wettbewerb. Wir haben es also auch bei der Komposition nur mit Basisfähigkeit für bestimmte Muster zu tun, und bei den über Basisfähigkeit definierten neuen Stufen WB-WORT und WB-SYNTAGMA mit Kategorien, die nicht nur auf Stammbildungsphänomene beschränkt sind, sondern diese auch nur sehr punktuell abdecken.

\subsection{Abweichungen im Hinblick auf syntaktische Kriterien}

Aufmerksame Leser ahnen schon, wie es weitergeht: Die Basisstufen WORT und SYNTAGMA, die inzwischen nur noch die Bewegungs- und Erweiterungseigenschaften LK-TEIL, VF-TEIL und E-TEIL festhalten, müssen noch weiter aufgespalten werden, wenn man an der bisherigen Strategie festhalten will. So haben nicht alle verbalen Komplexe mit einem Zweitglied, das in die linke Klammer bewegt werden kann, ein Erstglied, das man ins Vorfeld rücken kann. Vor allem bei Partikelverben divergieren diese Kriterien oft, vgl. (6)-(8) für aussprechen, einstellen und miederkehren. ${ }^{22}$

(6) a. Er sprach sich nicht gegen diese L ösung aus.

b. * Aus sprach er sich gegen diese Lösung nicht.

$21 \quad$ Vgl. z.B. Meibauer (2003).

22 Die Akzeptabilitätsmarkierungen beruhen auf entsprechenden Informantenbefragungen. 
(7) a. Wir stellen Bewerber erst nach Absolvierung eines Eignungstests ein.

b. ??Ein stellen wir Bewerber erst nach Absolvierung eines Eignungstests.

(8) a. Eine Krise dieses Ausmaßes kebrt nicht so schnell wieder.

b. * Wieder kehrt eine Krise dieses Ausmaßes nicht so schnell.

Nun könnte man vermuten, dass es unabhängige Gründe für die geringe Akzeptabilität der b-Beispiele gibt. Dann wäre das Kriterium VF-TEIL für die fraglichen Verben möglicherweise gar nicht einschlägig, und ihre Einstufung als Syntagmen würde nicht zu einem Widerspruch mit anderen Kriterien führen. ${ }^{23}$

Wir haben in dem erwähnten Forschungsprojekt nach möglichen unabhängigen Gründen für die Nicht-Vorfeldfähigkeit der Erstglieder vieler Partikelverben gesucht, wurden aber nicht fündig. Naheliegende Vermutungen, etwa Blockaden wegen fehlender Kontrastfähigkeit oder mangelnder semantischer Transparenz, erwiesen sich als nicht stichhaltig, vgl. Heine/Jacobs/ Külpmann (2010).

Dass man fehlende Vorfeldfähigkeit in Fällen wie in (6b)-(8b) nicht auf solche Faktoren zurückführen kann, zeigt sich auch darin, dass es viele funktional ähnliche Partikelverben gibt, bei denen die beiden Bewegungskriterien nicht divergieren. Man betrachte die auf Korpusbelegen beruhenden Beispiele (9)-(11):

(9) a. Es fing alles am 2. Januar 1889 an.

b. An fing alles am 2. Januar 1889.

(10) a. Es fällt auf, dass im Schreiben der Stadtverwaltung ausdrücklich darauf hingewiesen wurde, dass ...

b. Auf fällt, dass im Schreiben der Stadtverwaltung von gestern ausdrücklich darauf hingewiesen wurde, dass ...

(11) a. Es geht schon nächste Woche Los.

b. Los geht es schon nächste Woche.

Hier haben wir es mit semantisch nicht-transparenten Verben zu tun, und in naheliegenden Verwendungskontexten wird weder das Verb noch sein Erstglied mit anderen Elementen kontrastiert. Dennoch sind die b-Varianten von (9)-(11) deutlich akzeptabler als die von (6)-(8).

Das Kriterium der Vorfeldfähigkeit scheint also für Partikelverben durchaus einschlägig zu sein, muss dann aber wegen Fällen wie (6)-(8) im Stufensystem von dem anderen Bewegungskriterium LK-TEIL getrennt werden.

Und leider muss man VF-TEIL auch von dem Kriterium E-TEIL trennen, denn nicht alles, was ins Vorfeld bewegt werden kann, erlaubt auch Modifikation, Komplementierung etc. Das zeigt sich an Beispielen wie (12)-(13):

23 Vgl. die Bemerkungen in Kapitel 2 zum Umgang mit nicht einschlägigen Kriterien. 
(12) a. dass dieses Verfahren dann [sehr schnell [Schule machte]]

b. ??dass dieses Verfahren dann [sehr schnelle Schule] machte

c. Schule machte dieses Verfabren dann sebr schnell.

(13) a. dass er im Streitpunkt Studiengebühren [deutlich [Farbe bekennen]] muss

b. * dass er im Streitpunkt Studiengebübren [deutliche Farbe] bekennen muss

c. Farbe bekannte er auch im Streitpunkt Studiengebübren.

Die nominalen Erstglieder von Schule machen und Farbe bekennen können nicht durch ein Adjektiv modifiziert werden, obwohl sie ins Vorfeld bewegt werden können. Das kann man übrigens nicht mit der mangelnden Referentialität dieser Erstglieder erklären. Nicht-referenzielle Nomina können in anderen Fällen durchaus modifiziert werden, vor allem, wenn der Modifikator, wie in (12)-(13), Skopus über den ganzen Komplex aus $N$ und $V$ hat. ${ }^{24}$

Wir landen nun also bei dem Stufensystem in Tabelle 6 und bei Klassifikationen wie in (14):

\begin{tabular}{|c|c|c|}
\hline & LK-WORT & LK-SYNTAGMA \\
\hline \multirow[t]{2}{*}{ LK-TEIL } & - & + \\
\hline & VF-WORT & VF-SYNTAGMA \\
\hline \multirow[t]{2}{*}{ VF-TEIL } & - & + \\
\hline & E-WORT & E-SYNTAGMA \\
\hline \multirow[t]{2}{*}{ E-TEIL } & - & + \\
\hline & P-WORT & P-SYNTAGMA \\
\hline DO-SYLB & + & - \\
\hline DO-PHONOTAKT & + & - \\
\hline \multirow[t]{2}{*}{ DO-MINPHON } & + & - \\
\hline & G-WORT & G-SYNTAGMA \\
\hline INTERN-SPAT & - & + \\
\hline INTERN-MAJUSK & - & + \\
\hline \multirow[t]{2}{*}{ DO-MINGRAPH } & + & - \\
\hline & F-WORT & F-SYNTAGMA \\
\hline \multirow[t]{2}{*}{ FLEX-TEIL } & - & + \\
\hline & WB-WORT & WB-SYNTAGMA \\
\hline DERIV-BAS & + & - \\
\hline KOMP-BAS & + & - \\
\hline
\end{tabular}

Tab. 6: System 6

$24 \quad$ Vgl. z.B. dass sie tief/ tiefen Atem holen musste. 
(14) a. LK-WORT, VF-WORT, E-WORT, P-WORT, G-WORT, F-WORT, WB-WORT: sprechen, befragen, Frage, ...

b. LK-WORT, VF-WORT, E-WORT, P-SYNTAGMA, G-WORT, F-WORT, WB-WORT:

Bahnkunde, blutrot, ...

c. LK-WORT, VF-WORT, E-WORT, P-SYNTAGMA, G-WORT, F-SYNTAGMA, WB-WORT: ${ }^{25}$ rïbrbraten, Hohepriester, ...

d. LK-SYNTAGMA, VF-WORT, E-WORT, P-SYNTAGMA, G-WORT, F-SYNTAGMA, WB-WORT:

absprechen, ausprechen, einstellen, ...

e. LK-SYNTAGMA, VF-SNYTAGMA, E-WORT, P-SYNTAGMA, G-WORT, F-SYNTAGMA, WB-SYNTAGMA:

anfangen, gefangen nehmen, kennenlemen, ...

f. LK-SYNTAGMA, VF-SYNTAGMA, E-WORT, P-SYNTAGMA, G-SYNTAGMA, F-SYNTAGMA, WB-SYNTAGMA:

Farbe bekennen, Schule machen, ...

g. LK-SYNTAGMA, VF-SYNTAGMA, E-SYNTAGMA, P-SYNTAGMA, G-SYNTAGMA, F-SYNTAGMA, WB-SYNTAGMA: schnell sprechen, Gerda befragen, viele schwienige Fragen, ...

Hier wird nun endgültig das Dilemma der Aufspaltungsstrategie deutlich: Wenn man alles widerspruchsfrei, vollständig und im Detail erfassen will, wird die Zahl der Stufen annähernd so groß wie die der grammatischen Eigenschaften, die durch sie festgehalten werden sollen. Dabei ist das Bild, das System 6 zeichnet, noch zu schön: Man erinnere sich, dass man auch das P-WORT und das G-WORT weiter aufspalten muss, um internen Divergenzen in der Phonologie bzw. Graphematik Rechnung zu tragen (vgl. 3.1 bzw. 3.2). Damit hätte man neun Stufenpaare zur Kodierung von zwölf Eigenschaftsdimensionen. Und es geht weiter in Richtung eines Eins-zu-EinsVerhältnisses zwischen Stufen und Eigenschaften, wenn man einige der Kriterien genauer betrachtet. Dann zeigt sich z.B., dass das Kriterium E-TEIL in drei Kriterien aufgespalten werden muss, da Koordinierbarkeit, Modifizierbarkeit/Komplementierbarkeit und Determinierbarkeit divergieren können. ${ }^{26}$ Und sogar das Vorfeldkriterium VF-TEIL muss differenziert werden, da die Vorfeldfähigkeit in Fällen wie (9)-(11) nur eine bedingte ist, nämlich voraussetzt, dass das zur Vorfeldpartikel gehörende verbale Zweitglied un-

25 Ob diese Beispiele WB-Wörter sind, ist nicht ganz sicher. Eine genauere Klärung muss hier jedoch unterbleiben.

26 Vgl. für Modifizierbarkeit vs. Determinierbarkeit z.B. Tiefen Atem bolen vs. ${ }^{*}$ Einen Atem bolen. 
mittelbar in der linken Satzklammer folgt. ${ }^{27}$ Die Verteilung dieser weiter differenzierten Kriterien auf die Daten ist so, dass wieder neue Stufenpaare nötig werden. Dann hätten wir 12 Stufenpaare zur Kodierung von 14 Eigenschaftsdimensionen, d.h. jede einzelne Stufe würde im Durchschnitt weniger als 1,2 Eigenschaften voraussagen.

\section{Alternativen zur Aufspaltung des Stufensystems}

Ich fasse zusammen: Die ursprünglich mit den Ausdrucksstufen WORT und SYNTAGMA verbundene Annahme, man könne mit wenigen Kategorien die Ausdrücke der Sprache vollständig erfassen und dabei auf ökonomische Weise viele ihrer Details festhalten (vgl. 2.), scheitert an der Differenziertheit der Daten. Wenn man dieses Problem, in Anlehnung an Vorschläge aus der Forschung, durch eine Aufspaltung des Stufensystems zu lösen versucht, bekommt man eine große Zahl von Stufen, die jeweils kaum mehr als eine einzige Eigenschaft voraussagen. Bei dieser Strategie erweisen sich die Ausdrucksstufen also nicht, wie erhofft, als reiche Kategorien, sondern als sehr arme, die keinen erkennbaren Mehrwert gegenüber einer Beschreibung haben, die auf sie verzichtet. Welchen Vorteil hat z.B. die Einordnung eines Ausdrucks in die Kategorie F-WORT gegenüber einer, die ihn als Ausdruck ohne separat flektierbare Teile klassifiziert? Offensichtlich keinen, wenn F-WORT eben nichts anderes bedeutet als, Ausdruck ohne separat flektierbare Teile? Es wäre sogar unökonomisch, zusätzlich zur Kategorie AUSDRUCK OHNE SEPARAT FLEKTIERBARE TEILE noch eine Kategorie F-WORT anzunehmen, wenn beide völlig deckungsgleich sind.

\subsection{WORT und SYNTAGMA als Prototypenkategorien}

Aber gibt es nicht Alternativen zur Aufspaltungsstrategie, die es erlauben, an den ursprünglichen reichen Kategorien WORT und SYNTAGMA festzuhalten und trotzdem allen Daten widerspruchsfrei Rechnung zu tragen? Ein naheliegender Vorschlag wäre die Umdeutung der Stufen WORT und SYNTAGMA als Prototypen-Kategorien. Dann müsste ein Ausdruck, um als Wort zu gelten, nicht alle in System 1 genannten Eigenschaften haben, sondern nur so viele, dass er idealen Wörtern hinreichend ähnlich ist. Problemfälle wie Komposita oder Partikelverben könnten dann ohne Widersprüche in die Kategorie WORT eingeordnet werden, aber eben, weil sie nicht alle Eigenschaften idealer Fälle haben, nicht in das Zentrum, sondern in die Peripherie dieser Kategorie.

27 Vgl. z.B. (9b) vs. * An hat alles am 2. Jamuar gefangen. Näheres in Heine/Jacobs/Külpmann (2010). 
Dass sich diese Lösung in der Forschungspraxis nicht durchgesetzt hat, ${ }^{28}$ hat teilweise ideologische Gründe, ist aber aus der EAS-Perspektive durchaus rational. Wenn man Ausdrücke wie Babnkunde oder einstellen detailliert analysieren will, kann man es ja nicht bei der Feststellung belassen, sie seien periphere Wörter. Man muss vielmehr sagen, welche Worteigenschaften sie haben und welche nicht, z.B. dass sie aus zwei Syllabierungsdomänen bestehen, keine internen Spatien haben, dass Teile von einstellen, aber nicht von Babnkinde für syntaktische Prozesse zugänglich sind usw. Eine solche detaillierte und differenzierende Beschreibung folgt einfach nicht aus der Einordnung als peripheres Wort.

Das gilt übrigens auch, wenn man das $\mathrm{Maß}$ der Peripheralität nichtidealer Fälle komparativ oder quantitativ genauer spezifiziert, etwa nach der Zahl der Eigenschaften, die sie mit idealen Fällen gemeinsam haben. ${ }^{29}$ Auch wenn wir wissen, dass Babnkunde und einstellen zum Grad 0,8 bzw. 0,65 Wörter sind, wissen wir noch lange nicht, welche Worteigenschaften sie haben und durch welche sie sich unterscheiden.

\subsection{WORT und SYNTAGMA als Defaultkategorien}

Es gibt aber andere flexible Kategorisierungsstechniken, mit denen man auch nicht-ideale Fälle den ursprünglichen Kategorien WORT und SYNTAGMA subsumieren kann, ohne die Details unter den Tisch zu kehren. So kann man diese Kategorien als oberste Elemente eines Typsystems mit Default-Vererbung betrachten, in dem untergeordnete Typen von den übergeordneten nur die Eigenschaften übernehmen, die bei ihnen nicht ausdrücklich anders spezifiziert sind. ${ }^{30}$ Zudem kann ein untergeordneter Typ Eigenschaften mehrerer übergeordneter Typen erben, wenn das nicht zu widersprüchlichen Eigenschaftskombinationen führt. In einem solchen Kategorisierungssystem würden z.B. die Ausdrücke in (14a) und in (14g) genau die Eigenschaften der Kategorien WORT bzw. SYNTAGMA erben, die Ausdrücke in b-f jeweils nur die Teilmenge der Eigenschaften von WORT und SYNTAGMA, die bei ihnen nicht anders spezifiziert sind, wobei es zu einer Mischung von WORT- und SYNTAGMA-Eigenschaften käme, ohne dass die Stufen dazu aufgespalten werden müssen.

${ }_{28} \quad$ Fuhrhop (2007) stellt ihrer Arbeit zwar ein Bekenntnis zur Prototypentheorie voran (ebd., Kap. 1.2), ihre konkreten Untersuchungen zielen aber eher darauf ab, Problemfälle klassischen Kategorien zuzuordnen (etwa wenn sie fragt, ob rad in radfabren ein selbstständiges Substantiv, ein substantivisches Erstglied oder eine Verbpartikel ist; ebd., S. 55).

29 Ágel (2004) stellt eine auf der Zahl der zutreffenden Worteigenschaften beruhende Methode zur Ermittlung des quantitativen Grads der ,Wortigkeit “ nicht-idealer Fälle vor. Aarts (2004) möchte mit einem ähnlichen Verfahren generell die komparative Peripheralität von Grenzfällen bestimmen.

30 Vgl. z.B. Müller (2007, Kap. 7.5.1). 
Aber auch bei dieser Modellierung hätten die Ausdrucksstufen kaum noch prädiktive Kraft. So könnte man daraus, dass ein Ausdruck X in der Vererbungshierarchie unter der Stufe WORT steht, noch keine einzige Eigenschaft von $X$ sicher voraussagen. Man müsste ja stets zusätzlich prüfen, welche WORT-Eigenschaften X erbt und welche nicht. Während also bei der Aufspaltungsstrategie die Aussagekraft der Stufen dadurch schwindet, dass sie kaum mehr als eine Eigenschaft vorhersagen, geschieht das bei der Default-Strategie dadurch, dass die Zuordnung zu einer Stufe allein nicht ausreicht, um die konkreten Eigenschaften eines Ausdrucks vorherzusagen. ${ }^{31}$

Eine weitere Strategie zur Rettung der traditionellen Ausdrucksstufen sei hier nur noch kurz erwähnt: Duale Analysen, wie sie für Partikelverben (unter sehr verschiedenen theoretischen Prämissen) von Goldberg (2003), Zeller (2003) und Kremers (2009) vorgeschlagen wurden, postulieren, dass ein und derselbe Ausdruck hinsichtlich bestimmter Aspekte und/oder in bestimmten Konstruktionen als Wort, hinsichtlich anderer Aspekte bzw. Konstruktionen als Syntagma einzustufen ist. Im Hinblick auf die Prädiktivität der Ausdrucksstufen hat das den gleichen unerwünschten Effekt wie die Default-Strategie: Aus den Ausdrucksstufen allein lassen sich keine sicheren Aussagen darüber mehr ableiten, welche der mit ihnen assoziierten Eigenschaften in konkreten Fällen tatsächlich vorliegen.

\subsection{Verzicht auf WORT und SYNTAGMA als metasprachliche Kategorien: Grammatik ohne Wörter}

Angesichts dieser Probleme sollte man durchaus erwägen, auf die traditionellen Ausdrucksstufen WORT und SYNTAGMA bzw. auf differenzierte oder aufgeweichte Versionen dieser Stufen ganz zu verzichten. Man könnte ja für alle Ausdrücke spezifizieren, welchen Wert die Eigenschaftsdimensionen DO-SYLB, INTERN-SPAT, FLEX-TEIL, LK-TEIL usw. bei ihnen annehmen, ohne zu versuchen, diese Eigenschaften zu Ausdrucksstufen zusammenzufassen. Dazu muss man diese Eigenschaften nicht für jeden Ausdruck einzeln notieren. Die allermeisten Ausdrücke folgen ja Mustern oder Regeln, an denen man die fraglichen Eigenschaften festmachen könnte. So muss man die spezifische Werteverteilung der Eigenschaftsdimensionen bei $\mathrm{N}+\mathrm{N}$-Determinativkomposita nicht bei jedem einzelnen Kompositum festhalten (was gar nicht möglich ist, die Klasse ist ja offen), sondern kann sie als Charakteristikum des Musters der $\mathrm{N}+\mathrm{N}$-Komposition repräsentieren.

31 Die Herausgeber des vorliegenden Bandes merkten hier an, dass die Default-Kategorisierung gegenüber der Aufspaltungsstrategie den Vorteil habe, zum Ausdruck zu bringen, dass bestimmte Stufeneigenschaften dazu neigen, zu kookkurrieren. $\mathrm{Zu}$ der Frage, ob das wirklich ein Vorteil ist, vgl. die in 4.3 folgenden Überlegungen zur Erfassung von Abhängigkeiten zwischen den Stufeneigenschaften. 
Im Hinblick auf Vollständigkeit, Detailliertheit und Widerspruchsfreiheit wäre eine solche Grammatike ohne Wörter der Prototypenstrategie überlegen und der Aufspaltungs- und Defaultstrategie ebenbürtig. Im Hinblick auf Beschreibungsökonomie hätte sie nur gegenüber der Defaultstrategie einen gewissen Nachteil, nämlich bei den Ausdrücken, die die ideale Verteilung der Eigenschaften aufweisen und deswegen bei dieser Strategie ohne weitere Angaben als WORT bzw. SYNTAGMA eingestuft werden können.

Einen Vorteil gegenüber allen anderen Strategien sehe ich darin, dass der Verzicht darauf, die Eigenschaften von vorneherein zu größeren Gruppen zusammenzufassen, den Blick freimacht auf die tatsächlichen Zusammenhänge zwischen ihnen. Bei 12 binären Eigenschaftsdimensionen wären $2^{12}$ Werteverteilungen rechnerisch möglich. Die Zahl der in den Daten belegten Verteilungen scheint jedoch weit niedriger zu sein. ${ }^{32}$ Es muss also Abhängigkeiten zwischen den Dimensionen geben, die es zu identifizieren und zu erklären gilt. In (A1)-(A4) sind einige Hypothesen über solche Abhängigkeiten aufgeführt:

$$
\begin{aligned}
& \text { (A1) }+ \text { VF-TEIL } \rightarrow+\text { LK-TEIL } \\
& \text { (A2) }+ \text { DO-SYLB } \rightarrow \text { (- LK-TEIL } \&-\text { VF-TEIL } \&-\text { E-TEIL }) \\
& (\text { A3) }+ \text { E-TEIL } \rightarrow+\text { INTERN-SPAT } \\
& \text { (A4) }+ \text { DERIV-BAS } \rightarrow-\text { INTERN-SPAT }
\end{aligned}
$$

Ad (A1): Wie es scheint, setzt die Möglichkeit, einen Teil eines Komplexes $\mathrm{K}$ ins Vorfeld zu bewegen, voraus, dass $\mathrm{K}$ einen in die linke Satzklammer bewegbaren verbalen Teilausdruck enthält. So können die Erstglieder mancher Partikelverben ins Vorfeld bewegt werden (siehe 3.4), nie jedoch die Erstglieder von Präfixverben, die keinen beweglichen verbalen Teil enthalten. ${ }^{33}$

\footnotetext{
$32 \quad$ Sie ist jedoch sicher größer als die sechs in (14) belegten Verteilungen. Wesentlich wächst die Zahl der belegten Kombinationen von Stufeneigenschaften zudem, wenn man die hier ausgeblendeten Eigenschaftsdimensionen (siehe Kap. 2) und die innerhalb der betrachteten Eigenschaftsdimensionen erforderlichen Differenzierungen (etwa beim Kriterium E-TEIL, 3.4) berücksichtigt. Nach bisherigen Ergebnissen unseres Projekts wächst damit die Zahl der belegten Kombinationen auf deutlich über 20. - Damit wird übrigens eine weitere denkbare Klassifizie rungsstrategie unplausibel, nämlich die, jede einzelne belegte Kombination zu einer Stufe zusammenzufassen. Ein Klassifizierungssystem mit über 20 sich ausschließenden Stufen widerspricht den in Kapitel 2 dargelegten Grundgedanken der Annahme von Ausdrucksstufen nicht weniger als das in Tabelle 6 dargestellte System mit sieben sich überkreuzenden Stufenpaaren.

33 Außerdem macht (A1) die Voraussage, dass Teilausdrücke von Komplexen, die überhaupt kein Verb enthalten, nie ins Vorfeld bewegt werden können. Das berührt die altbekannte Problematik allgemeiner Beschränkungen für Bewegungsprozesse, im Hinblick auf die (A1) präzisiert werden müsste. Das würde hier jedoch den Rahmen sprengen.
} 
Ad (A2): Ein Ausdruck, der eine Syllabierungsdomäne ist, hat nie bewegliche oder syntaktisch erweiterbare Teile. Anders gesagt: Um für interne syntaktische Prozesse offen zu sein, müssen Ausdrücke aus mehr als einer Syllabierungsdomäne bestehen. ${ }^{34}$

Ad (A3): Komplexe mit syntaktisch erweiterbaren Teilausdrücken werden getrennt geschrieben. ${ }^{35}$ Das ist insofern bemerkenswert, als, wie schon in 3.2 bemerkt, die Zugänglichkeit von Teilausdrücken für syntaktische Prozesse ansonsten für die Getrennt-/Zusammenschreibung irrelevant ist.

Ad (A4): Komplexe, die als Basen für relevante Derivationsprozesse, etwa Adjektivierung mit -bar, fungieren können, scheinen stets zusammengeschrieben zu werden. ${ }^{36}$

In dem Maß, wie solche Abhängigkeiten gesichert werden können, können sie im Rahmen einer Grammatik ohne Wörter auch zu einer ökonomischeren Darstellung der einschlägigen Eigenschaften herangezogen werden. Wenn sich etwa (A2) bestätigt, kann man sich darauf beschränken, diesen Zusammenhang einmal in der Grammatik festzuhalten, und muss die Unzugänglichkeit für interne syntaktische Prozesse nicht bei jedem einzelnen der vielen Ausdrücke, die nur eine Syllabierungsdomäne umfassen, wiederholen. Zusammen mit der schon erwähnten Möglichkeit, die fraglichen Eigenschaften an Mustern oder Regeln festzumachen (siehe oben), lässt das hoffen, dass eine Grammatik ohne Wörter weit übersichtlicher sein könnte, als man zunächst vermuten würde.

Darüber hinaus würde ein solcher Rahmen die hier diskutierten Eigenschaften in eine wesentlich reichere deduktive Struktur ${ }^{37}$ einbinden als alle Strategien, die an einem Kategorisierungssystem festhalten, das Teilmengen der Eigenschaften zu Ausdrucksstufen zusammenfasst. Diese Strategien haben außerdem, im Gegensatz zu der skizzierten Grammatik ohne Wörter, das empirische Problem, dass sie bidirektionale Abhängigkeiten zwischen den jeweils zusammengefassten Eigenschaften erwarten lassen, nämlich eben die Kookkurrenz der Eigenschaften. Zusammenhänge wie (A1)-(A4) gelten jedoch anscheinend immer nur unidirektional. Keine der folgenden bidirektionalen Varianten von (A1)-(A4) trifft zu:

34 Das ist eine Fassung des Prinzips der, lexikalischen Integrität ${ }^{c}$ (vgl. Kap. 2), die dessen Geltungsbereich gegenüber Fassungen, die auf dem Wortbegriff beruhen, erheblich einengt.

35 Vgl. Jacobs (2005, Kap. 4.1.4.2), wo auch einige wenige Gegenbeispiele zu dieser Implikation diskutiert werden.

36 Das gilt mit der Einschränkung, dass manche Resultativkomplexe, wie klein schneiden, de ren Adjektivierung mit bar nach den Untersuchungen in unserem Forschungsprojekt recht akzeptabel ist, erst in der neuesten Fassung der deutschen Orthographie zusammengeschrieben werden können.

${ }^{37}$ Der Reichtum der deduktiven Struktur erhöht sich noch dadurch, dass einige der festgestellten Abhängigkeiten wohl aus noch allgemeineren Zusammenhängen folgen, etwa (A1) aus Beschränkungen für Bewegungsprozesse. 
$\left(\mathrm{A} 1^{\prime}\right)+$ VF-TEIL $\leftrightarrow+$ LK-TEIL

$\left(\mathrm{A} 2^{\prime}\right)+$ DO-SYLB $\leftrightarrow(-$ LK-TEIL $\&-$ VF-TEIL $\&-$ E-TEIL $)$

$\left(\mathrm{A} 3^{\prime}\right)+$ E-TEIL $\leftrightarrow+$ INTERN-SPAT

$\left(4^{\prime}\right)+$ DERIV-BAS $\leftrightarrow-$ INTERN-SPAT

(A1') ist falsch, weil es unter Partikelverben (vgl. 3.4.), Inkorporationsbildungen und Rückbildungen, aber auch unter Komplexen der Form Partikel + Verb (wie nicht qustimmen) viele ohne vorfeldfähiges Erstglied, aber mit beweglichem verbalen Zweitglied gibt. (A2') ist falsch, weil es viele Ausdrücke gibt, die mehr als eine Syllabierungsdomäne umfassen, aber syntaktisch inert sind (z.B. sämtliche Komposita). (A3') ist falsch, weil Getrenntschreibung auch bei Komplexen möglich ist, die keine syntaktisch erweiterbaren Teile haben, so bei manchen Zusammenrückungen, z.B. <an Stelle>, <auf Grund>, < bei weitem>. Und (A4') trifft nicht zu, weil es zusammengeschriebene Komplexe gibt, die nicht als Basis für relevante Derivationsprozesse, etwa -bar-Adjektivierung, fungieren können, z.B., wie schon in 3.3 gesagt, Inkorporationsbildungen wie bloßstellen.

Dieser Nachteil der Zusammenfassung der Eigenschaften zu Ausdrucksstufen mag vermeidbar erscheinen, wenn man sich damit begnügt, die Eigenschaften, die mit einer gewissen Wabrscheinlichkeit kookkurrieren, anzugeben. So könnte man die Subsumierung der Eigenschaften unter die Kategorie WORT einsetzen, um festzuhalten, dass ein Ausdruck, der, sagen wir, die Eigenschaft - INTERN-SPAT hat, mit einer gewissen Wahrscheinlichkeit auch die Eigenschaften + DO-SYLB, - E-TEIL usw. hat. (Eine solche probabilistische Deutung der Ausdrucksstufen liegt auch nahe, wenn man sie als Default-Kategorien betrachtet, vgl. 4.2) Ein Problem bei dieser Sicht ist allerdings, dass für die Kookkurrenz der verschiedenen Eigenschaften in der Regel unterschiedliche Wahrscheinlichkeiten gelten. So kookkurriert - INTERN-SPAT mit hoher Wahrscheinlichkeit mit - E-TEIL, während die Kookkurrenz mit + DO-SYLB angesichts der Unzahl der zusammengeschriebenen Komposita sicher weniger wahrscheinlich ist. Solche Unterschiede könnte man auch im Rahmen einer probabilistischen Deutung der Stufenkategorien nur durch eine Aufspaltung dieser Kategorien erfassen - und geriete letztlich wieder in das in Kapitel 3 beschriebene Dilemma.

\section{Was wird aus den stufenbezogenen Kompartmentalisierungen?}

Mit den klassischen Ausdrucksstufen gerät auch die auf ihnen beruhende Kompartmentalisierung der Grammatik in Morphologie vs. Syntax vs. Phraseologie ins Wanken. Egal, ob man die Stufen in viele spezifischere Katego- 
rien aufspaltet, sie nur noch in abgeschwächten Versionen oder überhaupt nicht mehr verwendet: Man wird die Idee, dass sich die Organisation unseres sprachlichen Wissens an der Unterscheidung WORT vs. SYNTAGMA orientiert, entweder ganz aufgeben oder zumindest so stark differenzieren müssen, dass die ursprüngliche Hoffnung, man könne auf dieser Grundlage ein einfaches Bild von der Architektur von Sprachsystemen zeichnen, auf jeden Fall aufgegeben werden muss. Das betrifft auch die Abgrenzung des Lexikons von der Grammatik, soweit diese auf der Unterscheidung von Wörtern und Syntagmen beruht (vgl. Kap. 1).

Dieser Verlust an Übersichtlichkeit wird jedoch mehr als kompensiert durch einen Zugewinn an Realismus. Außerdem öffnen sich einer Linguistik, die sich von zu simplen Vorstellungen über die stufenbasierte Gliederung von Sprachsystemen verabschiedet, Wege zur Beendigung diverser verfahrener Abgrenzungsdebatten, etwa der über den Grenzverlauf zwischen einer nach dem Kriterium „Polylexikalität" identifizierten Phraseologie und einer auf „Monolexikalität" beruhenden Morphologie, die solche Begriffskrücken wie den „Einwortphraseologismus" hervorgebracht hat. ${ }^{38}$

\section{Wozu noch Wörter?}

Wir müssen uns also im Hinblick auf die Ausdrucksstufen und die auf ihnen basierenden Einteilungen des Sprachsystems von liebgewordenen Gewohnheiten verabschieden, wenn wir an den EAS-Zielen Vollständigkeit, Detailtreue und Widerspruchsfreiheit festhalten wollen. Aber natürlich orientieren sich Sprachwissenschaftler nicht immer an diesen Maximalzielen. So betrachten sie oft nicht die Gesamtheit der Ausdrücke einer Sprache, sondern nur bestimmte Teilklassen. Und dabei können dann die Abgrenzungsprobleme zwischen Wörtern und Syntagmen irrelevant werden. Wenn man z.B. - aus welchen Gründen auch immer - nur Ausdrücke betrachtet, die in Beispielreihe (14) in die Zeilen a oder g gehören, kann man ohne Ungereimtheiten mit den ursprünglichen Stufen WORT und SYNTAGMA arbeiten. Man kann diese Kategorien aber auch dann ohne Differenzierung oder Aufweichung verwenden, wenn man geringere Ansprüche an die Detailtreue stellt. Oft interessiert man sich z.B. als Phonologe nicht für syntaktische oder morphologische Eigenschaften, d.h. man betrachtet nur die ersten drei Eigenschaftsdimensionen in unserem ursprünglichen System (Tabelle 1). Wenn man das tut, kann man ohne Probleme alle Ausdrücke, die in (14) unter $\mathrm{b}$ bis $\mathrm{g}$ aufgeführt sind, als Syntagmen betrachten und muss sich nicht um Unterschiede etwa zwischen Nominalkomposita und Partikelverben kümmern. Und auch bei der Widerspruchsfreiheit machen wir oft Kompro-

38 Vgl. Ágel (2004), Heine (2010), Szczek (2004). 
misse. Wenn es z.B. nur um die grobe Zusammenfassung von Ausdrücken geht, die irgendwelche Worteigenschaften haben, aber nicht notwendigerweise alle und nicht alle dieselben, ist die Verwendung der Kategorie WORT trotz aller Abgrenzungsprobleme unschädlich. Und natürlich kann man Einschränkungen der Vollständigkeit mit solchen der Detailtreue oder der Widerspruchsfreiheit verbinden, und hat dann erst recht mit der Unterscheidung von WÖRTERN und SYNTAGMEN keine Probleme mehr.

Darin liegt wohl ein wesentlicher Grund dafür, dass sich diese Unterscheidung in der sprachwissenschaftlichen Praxis so hartnäckig hält. In dieser Praxis sind Einschränkungen der Vollständigkeit, der Detailtreue (oder ,Granularität) und der Widerspruchfreiheit ja gang und gäbe. Und das ist auch nicht generell zu kritisieren, sondern oft sehr sinnvoll, insbesondere vor dem Hintergrund bestimmter Anwendungsziele. Wenn man z.B. Lehrwerke für Deutsch als Fremdsprache entwirft, ist eine Einschränkung auf bestimmte gängige Ausdrucksklassen und die Wahl eines nicht allzu hohen Detailliertheitsgrades in der Regel unerlässlich. In einem solchen Arbeitskontext kann dann aus den genannten Gründen auch die Verwendung der traditionellen Unterscheidung von Wörtern und Syntagmen ungefährlich, ja sogar sehr praktisch sein.

Legitim kann der Einsatz dieser klassischen Begriffe auch sein, wenn man sie nicht als metasprachliche Kategorien im Rahmen der Sprachsystemanalyse, sondern in anderen Zusammenhängen verwendet. So bleibt es trotz - oder gerade wegen - der aufgezeigten Probleme eine interessante Frage, von welchem mentalen Konsept Grammatiker einer bestimmten Schule oder auch Laien einer bestimmten Sprachgemeinschaft ausgehen, wenn sie Ausdrücke als Wörter oder Nicht-Wörter klassifizieren. Interessant ist das etwa im Hinblick darauf, welche der in System 1 genannten Eigenschaftsdimensionen dieses mentale Wortkonzept am stärksten prägen. (Es ist oft vermutet worden, dass das die graphematischen Dimensionen sind, dass der Wortbegriff also wesentlich schriftinduziert ist.)

Kurz: Das Überleben der aus EAS-Sicht hochproblematischen Unterscheidung von Wörtern und Syntagmen erscheint nicht nur erklärlich, sondern sogar gerechtfertigt vor dem Hintergrund der Tatsache, dass die Sprachwissenschaft ein arbeitsteiliges Unterfangen mit unterschiedlichen methodischen Standards und unterschiedlichem Gegenstandsbezug ist. Entsprechendes gilt für die auf den klassischen Stufen aufbauende Abgrenzung von Syntax, Morphologie und Phraseologie, und auch für die von Lexikon und Grammatik. 


\section{Literatur}

Aarts, Bas (2004): Modelling linguistic gradience. In: Studies in Language 28, 1, S. 1-50.

Ágel, Vilmos (2004): Polylexikalität oder am Anfang waren mindestens zwei Wörter. Über eine Grundfrage (nicht nur) der Phraseologie. In: Brdar-Szabó, Rita/KnipfKomlósi, Elisabeth (Hg.): Lexikalische Semantik, Phraseologie und Lexikografie. Abgründe und Brücken. Festgabe für Regina Hessky. Frankfurt a.M., S. 21-50.

Croft, William (2001): Radical construction grammar. Syntactic theory in typological perspective. Oxford.

Dixon, Robert M.W./Aikhenvald, Alexandra Y. (Hg.) (2002): Word. A cross-linguistic typology. Cambridge.

Fuhrhop, Nanna (2007): Zwischen Wort und Syntagma. Zur grammatischen Fundierung der Getrennt- und Zusammenschreibung. (= Linguistische Arbeiten 513). Tübingen.

Fuhrhop, Nanna (2008): Das graphematische Wort (im Deutschen): Eine erste Annäherung. In: Zeitschrift für Sprachwissenschaft 27, 2, S. 189-228.

Goldberg, Adele E. (2003): Words by default: The Persian complex predicate construction. In: Francis, Elaine J./Michaelis, Laura A. (Hg.): Mismatch: Form-function incongruity and the architecture of grammar. Stanford, S. 117-146.

Hall, Tracy Alan (1999a): The phonological word: A review. In: Hall/Kleinhenz (Hg), S. $1-22$.

Hall, Tracy Alan (1999b): Phonotactics and the prosodic structure of German function words. In: Hall/Kleinhenz (Hg.), S. 99-132.

Hall, Tracy Alan/Kleinhenz, Ursula (Hg.) (1999): Studies on the phonological word. (= Current Issues in Linguistic Theory 174). Amsterdam.

Heine, Antje (2010): Wieviel Polylexikalität braucht ein Phraseologismus? In: Korhonen, Jarmo et al. (Hg): Europhras 2008. Beiträge zur internationalen Phraseologiekonferenz 2008 in Helsinki. Helsinki, S. 11-18.

Heine, Antje/Jacobs, Joachim/Külpmann, Robert (2010): Quer zu den Feldern - Zur Topologie von Partikelverben. In: Linguistische Berichte 221, S. 37-60.

Hockett, Charles F. (1944): Rezension von „Linguistic Interludes and Morphology: the descriptive analysis of words ${ }^{c c}$ von Eugene A. Nida. In: Language 20, S. 252-255.

Jacobs, Joachim (2005): Spatien - Zum System der Getrennt- und Zusammenschreibung im heutigen Deutsch. (= Linguistik - Impulse \& Tendenzen 8). Berlin/New York.

Kremers, Joost (2009): A prosodic approach to particle verbs. In: Linguistische Berichte 217, S. 81-96.

Meibauer, Jörg (2003): Phrasenkomposita zwischen Wortsyntax und Lexikon. In: Zeitschrift für Sprachwissenschaft 22, S. 153-188.

Müller, Stefan (2007): Head-driven phrase structure grammar. Eine Einführung. (= Stauffenburg Einführungen 17). Tübingen. 
Nespor, Marina/Vogel, Irene (1986): Prosodic phonology. (= Studies in Generative Grammar 28). Dordrecht u.a.

Primus, Beatrice (2003): Zum Silbenbegriff in der Schrift-, Laut- und Gebärdensprache. In: Zeitschrift für Sprachwissenschaft 22, 1, S. 3-55.

Nübling, Damaris et al. (2008): Historische Sprachwissenschaft des Deutschen. Eine Einführung in die Prinzipien des Sprachwandels. 2. Aufl. Tübingen.

Raffelsiefen, Renate (1999): Diagnostics for prosodic words revisited: The case of historically prefixed words in English. In: Hall/Kleinhenz (Hg.), S. 133-202.

Szczek, Joanna (2004): Einwortphraseologismen und ihr Verhältnis zur Phraseologie (am Beispiel des Deutschen und des Polnischen). In: Földes, Csaba/Wirrer, Jan (Hg): Phraseologismen als Gegenstand sprach- und kulturwissenschaftlicher Forschung. Baltmannsweiler, S. 75-85.

Wurzel, Wolfgang Ulrich (2000): Was ist ein Wort? In: Thieroff, Rolf et al. (Hg.): Deutsche Grammatik in Theorie und Praxis. Tübingen, S. 29-42.

Zeller, Jochen (2003): Moved preverbs in German: Displaced or misplaced? In: Booji, Geert/van Marle, Jaap (Hg.) (2003): Yearbook of Morphology 2003. Dordrecht, S. 179-212. 